\title{
A NEW APPROACH FOR SOLVING NONLINEAR VOLTERRA INTEGRO-DIFFERENTIAL EQUATIONS WITH MITTAG-LEFFLER KERNEL
}

\author{
ROGHAYEH MOALLEM GANJI AND HOSSEIN JAFARI
}

\begin{abstract}
In this work, we consider a general class of nonlinear Volterra integro-differential equations with Atangana-Baleanu derivative. We use the operational matrices based on the shifted Legendre polynomials to obtain numerical solution of the considered equations. By approximating the unknown function and its derivative in terms of the shifted Legendre polynomials and substituting these approximations into the original equation and using the collocation points, the original equation is reduced to a system of nonlinear algebraic equations. An error estimate of the numerical solution is proved. Finally, some examples are included to show the accuracy and validity of the proposed method.
\end{abstract}

\section{Introduction}

In recent years, several definitions for fixed and variable order fractional integral and derivative operators have been introduced, such as Riemann-Liouville [7, 15, 30], Grünwald-Letnikov [30], Caputo [30], Caputo and Fabrizio [3], Atangana and Baleanu (AB) [1] (to see more, refer to $[6,15,30,27,31]$ ).

Many problems in different fields of science and engineering such as viscoelasticity and damping, diffusion and wave propagation and chaos can be modeled by fractional derivative and integral operators $[4,5,13,15,19,26,28,29,31]$. For example, Pedro et al. [23] have investigated the drag force acting on a particle due to the oscillatory flow of a viscous fluid.

Fractional integro-differential equations are used in different applications of physics, chemistry, biology, engineering and applied mathematics. For example, Zakes and Sniady in [32] have studied the dynamic behavior of multispan uniform continuous beam arbitrarily supported on its edges subjected to various types of moving noninertial loads. Li et al. have used differential equations to model a memory behavior of shape-memory polymer [16].

In most cases, It is not easy to obtain exact solution of most these equations. Therefore, some researchers have proposed several approximation and numerical methods for solving such equations. For example, Rakhshan and Effati applied a generalized Legendre-Gauss collocation method for solving nonlinear fractional

2010 Mathematics Subject Classification. 42B25, 31C15.

Key words and phrases. Volterra integro-differential equations, Atangana-Baleanu derivative, Shifted Legendre polynomials, Operational matrix, Collocation points. 
differential equations [24]. Ganji et al. applied the fifth-kind Chebyshev polynomials to obtain solution variable orders differential equations [12]. Zhao and Wang solved space-time fractional partial differential equations using fast finite difference methods [33]. Liu et al. used a preconditioned fast quadratic spline collocation method for partial differential equations [17]. In [25], authors solved Fredholm integral equations by the reproducing kernel Hilbert space method. A numerical scheme based on the Bernstein polynomials for solving diffusion-wave equations has been proposed in [11] (to see more, refer to [7, 8, 14, 21]).

In recent years, by increasing number of applications of fractional integrodifferential equations, researchers attention have been given to use of orthogonal basis functions. The concept of operational matrices of these basis functions can be employed for the solution of the problems [20]. Recently, many researchers applied orthogonal basis functions for approximating. For example, Liu et al. solved variable order fractional differential-integral equations using Chebyshev polynomials [18]. Nemati et al. applied Legendre polynomials for solving twodimensional nonlinear Volterra integral equations [22]. In [9] and [10], Jacobi polynomials for solving differential equations of variable order have been proposed. By using these basis functions and their operational matrices, the main problem reduces to an algebraic system, which can be solved. Then, the approximate solution can be calculated.

In this work, we study the following type of the nonlinear Volterra integrodifferential equations

$$
\begin{aligned}
& A B C D_{t}^{\omega} u(t)=f(t, u(t))+\lambda T u(t), \quad t \in[0,1], \\
& u(0)=u_{0},
\end{aligned}
$$

where

$$
T u(t)=\int_{0}^{t} K(t, s) \phi(s, u(s)) d s,
$$

and $0<\omega \leq 1$, the parameter $\lambda$ is a real constant, $K, \phi$ and $f$ are given known functions, $u(t)$ is an unknown function. ${ }^{A B C} D_{t}^{\omega}$ denotes the Caputo $\mathrm{AB}^{-}$ derivative operator.

The organization of this paper is as follows: in Section 2, we present basic definitions of fractional calculus. Section 3 is introduced the main properties of the shifted Legendre polynomials (SLPs) and operational matrices based on the SLPs. Section 4 is devoted to proposing a new numerical method for solving problem (1.1). In Section 5, an error estimate is proved for the numerical solution. Section 6 includes some illustrative examples. Finally, we conclude the paper in Section 7 .

\section{Basic definitions and theorems}

Definition 2.1 (See [30]). Let $0<\omega \leq 1$. The RL-integral is defined as

$$
R L I_{t}^{\omega} u(t)=\frac{1}{\Gamma(\omega)} \int_{0}^{t}(t-s)^{\omega-1} u(s) d s
$$


The RL-integral of order $\omega$ satisfies the following property

$$
{ }^{R L} I_{t}^{\omega} t^{v}=\frac{\Gamma(v+1)}{\Gamma(v+1+\omega)} t^{v+\omega}, \quad v \geq 0 .
$$

Definition 2.2 (See [30]). Let $0<\omega \leq 1, u \in H^{1}(0,1)$ and $A B(\omega)$ be a normalization function suchthat $A B(0)=A B(1)=1$ and $A B(\omega)=1-\omega+\frac{\omega}{\Gamma(\omega)}$. Then

(1) The Riemann AB-derivative is given as

$$
{ }^{A B R} D_{t}^{\omega} u(t)=\frac{A B(\omega)}{1-\omega} \frac{d}{d t} \int_{0}^{t} E_{\omega}\left(-\frac{\omega}{1-\omega}(t-s)^{\omega}\right) u(s) d s,
$$

where $E_{\omega}(t)=\sum_{i=0}^{\infty} \frac{t^{i}}{\Gamma(\omega i+1)}$ is the Mittag-Leffler function.

(2) The Caputo AB-derivative is defined as

$$
{ }^{A B C} D_{t}^{\omega} u(t)=\frac{A B(\omega)}{1-\omega} \int_{0}^{t} E_{\omega}\left(-\frac{\omega}{1-\omega}(t-s)^{\omega}\right) u^{\prime}(s) d s .
$$

(3) The AB-integral is given as

$$
{ }^{A B} I_{t}^{\omega} u(t)=\frac{1-\omega}{A B(\omega)} u(t)+\frac{\omega}{A B(\omega) \Gamma(\omega)} \int_{0}^{t}(t-s)^{\omega-1} u(s) d s .
$$

Let $\alpha_{\omega}=\frac{1-\omega}{A B(\omega)}$ and $\beta_{\omega}=\frac{1}{A B(\omega) \Gamma(\omega)}$, then we can rewrite (2.1) as

$$
{ }^{A B} I_{t}^{\omega} u(t)=\alpha_{\omega} u(t)+\beta_{\omega} \Gamma(\omega+1)^{R L} I_{t}^{\omega} u(t) .
$$

It is easy to report that the $\mathrm{AB}$-derivative and $\mathrm{AB}$-integral satisfy the following properties

(1) ${ }^{A B C} D_{t}^{\omega} C=0, \quad C \in \mathbb{R}$,

(2) ${ }^{A B C} D_{t}^{\omega} t^{v}=\frac{A B(\omega)}{1-\omega} \sum_{i=0}^{\infty} \frac{(-1)^{i} \omega^{i} \Gamma(v+1)}{(1-\omega)^{i} \Gamma(\omega i+v+1)} t^{\omega i+v}, \quad v \geq 0$,

(3) ${ }^{A B C} D_{t}^{\omega} u(t)={ }^{A B R} D_{t}^{\omega} u(t)-\frac{A B(\omega)}{1-\omega} u(0) E_{\omega}\left(-\frac{\omega}{1-\omega} t^{\omega}\right)$,

(4) ${ }^{A B} I_{t}^{\omega} C=C\left(\alpha_{\omega}+\beta_{\omega} t^{\omega}\right), \quad C \in \mathbb{R}$,

(5) ${ }^{A B} I_{t}^{\omega} t^{v}=t^{v}\left(\alpha_{\omega}+\beta_{\omega}(v+\omega+1) B(v+1, \omega+1) t^{\omega}\right)$, where $B(\cdot, \cdot)$ is the Beta function,

(6) ${ }^{A B} I_{t}^{\omega}\left({ }^{A B} I_{t}^{\nu} u(t)\right)={ }^{A B} I_{t}^{\nu}\left({ }^{A B} I^{\omega} u(t)\right)$,

(7) ${ }^{A B} I_{t}^{\omega}\left({ }^{A B C} D_{t}^{\omega} u(t)\right)=u(t)-u(0)$.

Theorem 2.1 (See [1]). Let $C[0,1]$ be the space of all continuous functions defined on $[0,1]$ and $f, g \in C[0,1]$. Then the following inequalities can be established

$$
\begin{aligned}
& \left\|{ }^{A B R} D_{t}^{\omega} f(t)-{ }^{A B R} D_{t}^{\omega} g(t)\right\|_{\infty} \leq \delta\|f(t)-g(t)\|_{\infty}, \\
& \left\|^{A B C} D_{t}^{\omega} f(t)-{ }^{A B C} D_{t}^{\omega} g(t)\right\|_{\infty} \leq \delta\|f(t)-g(t)\|_{\infty},
\end{aligned}
$$


where $\delta$ is a constant number.

Theorem 2.2. Suppose that $f$ and $g$ satisfy the assumptions of Theorem 2.1, then we have

$$
\left\|^{A B} I_{t}^{\omega} f(t)-{ }^{A B} I_{t}^{\omega} g(t)\right\|_{\infty} \leq \varepsilon\|f(t)-g(t)\|_{\infty},
$$

where $\varepsilon=\alpha_{\omega}+\beta_{\omega}$.

Proof. According to definition of the AB-integral, we have

$$
\begin{aligned}
\left\|^{A B} I_{t}^{\omega} f(t)-{ }^{A B} I_{t}^{\omega} g(t)\right\|_{\infty} & =\left\|{ }^{A B} I_{t}^{\omega}(f(t)-g(t))\right\|_{\infty} \\
& =\left\|\alpha_{\omega}(f(t)-g(t))+\beta_{\omega} \Gamma(\omega+1)^{R L} I_{t}^{\omega}(f(t)-g(t))\right\|_{\infty} \\
& \leq \alpha_{\omega}\|f(t)-g(t)\|_{\infty}+\beta_{\omega} \Gamma(\omega+1)\left\|^{R L} I_{t}^{\omega}(f(t)-g(t))\right\|_{\infty} \\
& \leq\left(\alpha_{\omega}+\beta_{\omega}\right)\|f(t)-g(t)\|_{\infty} .
\end{aligned}
$$

Taking $\varepsilon=\alpha_{\omega}+\beta_{\omega}$, the proof is complete.

Theorem 2.3. Let $0<\omega \leq 1$ and $g:[0,1] \times \mathbb{R} \rightarrow \mathbb{R}$ be a continuous function such that there exits $\zeta$ satisfying

$$
\left|f\left(g, u_{1}(t)\right)-f\left(g, u_{2}(t)\right)\right| \leq \zeta\left|u_{1}(t)-u_{2}(t)\right|,
$$

for all $u_{1}, u_{2} \in \mathbb{R}$. Then the initial value problem given by equation (1.1) has a unique solution on $C[0,1]$

(1) If $\left(\alpha_{\omega}+\beta_{\omega}\right) \zeta_{1}<1$, when $\lambda=0$,

(2) If $\left(\alpha_{\omega}+\beta_{\omega}\right)\left(\zeta_{1}+|\lambda| \zeta_{2} \zeta_{3}\right)<1$, when $\lambda \neq 0$.

Proof. Taking the AB-integral of both sides of equation (1.1) gets

$$
\begin{aligned}
u(t)= & { }^{A B} I_{t}^{\omega} f(t, u(t))+\lambda{ }^{A B} I_{t}^{\omega} T u(t)+u_{0} \\
= & \alpha_{\omega} f(t, u(t))+\beta_{\omega} \Gamma(\omega+1)^{R L} I_{t}^{\omega} f(t, u(t))+\lambda \alpha_{\omega} T u(t) \\
& \quad+\lambda \beta_{\omega} \Gamma(\omega+1)^{R L} I_{t}^{\omega} T u(t)+u_{0} .
\end{aligned}
$$

We define the operator $\mathfrak{N}: C[0,1] \rightarrow C[0,1]$ by

$$
\begin{aligned}
\mathfrak{N} u(t)=\alpha_{\omega} f(t, u(t))+\beta_{\omega} \Gamma(\omega+1)^{R L} I_{t}^{\omega} f(t, u(t))+\lambda \beta_{\omega} \Gamma(\omega+1)^{R L} I_{t}^{\omega} T u(t) & \\
& +\lambda \alpha_{\omega} T u(t)+C,
\end{aligned}
$$

where $c=u_{0}-\alpha_{\omega} f\left(0, u_{0}\right)$. By (2.5), finding a solution of equation (1.1) in $C[0,1]$ is equivalent to finding a fixed point of the operator $\mathfrak{N}$. For $u_{1}, u_{2} \in C[0,1]$ and all $t \in[0,1]$, we have

$$
\begin{aligned}
\left|\mathfrak{N} u_{1}(t)-\mathfrak{N} u_{2}(t)\right|=\mid \alpha_{\omega} & \left(f\left(t, u_{1}(t)\right)-f\left(t, u_{2}(t)\right)\right)+\lambda \alpha_{\omega} T\left(u_{1}(t)-u_{2}(t)\right) \\
& +\beta_{\omega} \Gamma(\omega+1)^{R L} I_{t}^{\omega}\left(f\left(t, u_{1}(t)\right)-f\left(t, u_{2}(t)\right)\right) \\
& +\lambda \beta_{\omega} \Gamma(\omega+1)^{R L} I_{t}^{\omega} T\left(u_{1}(t)-u_{2}(t)\right) \mid \\
\leq \alpha_{\omega} & f\left(t, u_{1}(t)\right)-f\left(t, u_{2}(t)\right)+|\lambda| \alpha_{\omega}\left|T\left(u_{1}(t)-u_{2}(t)\right)\right| \\
& +\left.\beta_{\omega} \Gamma(\omega+1)\right|^{R L} I_{t}^{\omega}\left(f\left(t, u_{1}(t)\right)-f\left(t, u_{2}(t)\right)\right) \mid \\
& +\left.|\lambda| \beta_{\omega} \Gamma(\omega+1)\right|^{R L} I_{t}^{\omega} T\left(u_{1}(t)-u_{2}(t)\right) \mid .
\end{aligned}
$$


Let

$$
\varsigma_{3}=\sup _{t, s \in[0,1]} K(t, s),
$$

and according to (2.4), we have

$$
\begin{aligned}
& \left|f\left(t, u_{1}(t)\right)-f\left(t, u_{2}(t)\right)\right| \leq \varsigma_{1}\left|u_{1}(t)-u_{2}(t)\right|, \\
& \left|\phi\left(t, u_{1}(t)\right)-\phi\left(t, u_{2}(t)\right)\right| \leq \varsigma_{2}\left|u_{1}(t)-u_{2}(t)\right| .
\end{aligned}
$$

In veiw of $(2.6),(2.7)$ and according to $T u(t)$, we have

$$
\begin{aligned}
\left|\mathfrak{N} u_{1}(t)-\mathfrak{N} u_{2}(t)\right| \leq & \alpha_{\omega \varsigma_{1}}\left|u_{1}(t)-u_{2}(t)\right|+\beta_{\omega} \varsigma_{1}\left|u_{1}(t)-u_{2}(t)\right| \\
& \quad+|\lambda| \alpha_{\omega} \varsigma_{2} \varsigma_{3}\left|u_{1}(t)-u_{2}(t)\right|+|\lambda| \beta_{\omega} \varsigma_{2} \varsigma_{3}\left|u_{1}(t)-u_{2}(t)\right| \\
= & \left(\alpha_{\omega}+\beta_{\omega}\right)\left(\varsigma_{1}+|\lambda| \varsigma_{2} \varsigma_{3}\right)\left\|u_{1}-u_{2}\right\|_{\infty} .
\end{aligned}
$$

Therefore the operator $\mathfrak{N}$ is a contraction. The statement follows from Banach's Fixed Point Theorem.

\section{The SLPs and operational matrices based on the SLPs}

3.1. The SLPs. The SLPs on $[0,1]$ consist the set of orthogonal functions. The analytic form of the SLP of degree $n$ is defined by

$$
\mathcal{L}_{0}(t)=1, \mathcal{L}_{1}(t)=2 t-1, \mathcal{L}_{n}(t)=\sum_{k=0}^{n} a_{n, k} t^{k},
$$

where

$$
a_{n, k}=\frac{(-1)^{n+k}(n+k) !}{(n-k) !(k !)^{2}} .
$$

For two arbitrary functions $f, g \in L^{2}[0,1]$ the inner product and norm are defined, respectively, by

$$
\begin{gathered}
\langle f, g\rangle=\int_{0}^{1} f(t) g(t) d t \\
\|f\|_{L}^{2}(0,1)=\sqrt{\langle f, f\rangle} .
\end{gathered}
$$

Any arbitrary function $u \in L^{2}[0,1]$ can be expaned using the SLPs as follows

$$
u(t)=\sum_{i=0}^{\infty} u_{i} \mathcal{L}_{i}(t)=U^{T} \varphi(t),
$$

where

$$
u_{i}=Q^{-1} \int_{0}^{1} u(t) \mathcal{L}_{i}(t) d t
$$

and

$$
Q=\left[q_{i j}\right] \quad i, j=0,1, \cdots, M,
$$

and the elements $q_{i j}$ given by

$$
q_{i j}= \begin{cases}\frac{1}{2 i+1}, & i=j \\ 0, & i \neq j\end{cases}
$$


The function $u(t)$ can be approximated by taking only the first $M+1$ terms in (3.2) as

$$
u(t) \simeq u_{M}(t)=\sum_{i=0}^{M} u_{i} \mathcal{L}_{i}(t)=U^{T} \mathfrak{L}(t),
$$

where $U=\left[u_{0}, u_{1}, \ldots, u_{M}\right]^{T}$ and

$$
\mathfrak{L}(t)=\left[\mathcal{L}_{0}(t), \mathcal{L}_{1}(t), \cdots, \mathcal{L}_{M}(t)\right]^{T} .
$$

Similarly, any function $K(t, s)$ in $L^{2}([0,1] \times[0,1])$ can be expanded in terms of the SLPs as

$$
K(t, s) \simeq \mathfrak{L}^{T}(t) \mathcal{K} \mathfrak{L}(s),
$$

where $\mathcal{K}$ is an $(M+1) \times(M+1)$ matrix as

$$
\mathcal{K}=\left[k_{i, j}\right], \quad i, j=0,1, \ldots, M,
$$

and the shifted Legendre coefficients $k_{i, j}$ are qiven by

$$
k_{i, j}=\frac{\langle\langle K(t, s), \mathfrak{L}(t)\rangle, \mathfrak{L}(s)\rangle}{\left\|\mathcal{L}_{i}(t)\right\|_{2}^{2}\left\|\mathcal{L}_{j}(s)\right\|_{2}^{2}}, \quad i, j=0,1, \ldots, M
$$

\subsection{Operational matrices of the SLPs.}

(1) The integration of the vectors $\mathfrak{L}(t)$ defined by (3.4) can be approximately given by

$$
\int_{0}^{t} \mathfrak{L}(s) d s \simeq P \mathfrak{L}(t),
$$

where $P$ is an $(M+1) \times(M+1)$ matrix which is called the operational matrix of integration based on the SLPs and is given by

$$
P=\frac{1}{2}\left[\begin{array}{ccccccc}
1 & 1 & 0 & \cdots & 0 & 0 & 0 \\
\frac{-1}{3} & 0 & \frac{1}{3} & \cdots & 0 & 0 & 0 \\
\vdots & \vdots & \vdots & \ddots & \vdots & \vdots & \vdots \\
0 & 0 & 0 & \cdots & \frac{-1}{2 M-1} & 0 & \frac{1}{2 M-1} \\
0 & 0 & 0 & \cdots & 0 & \frac{-1}{2 M+1} & 0
\end{array}\right]
$$

(2) It is needed to evaluate the product of $\mathfrak{L}(t)$ and $\mathfrak{L}^{T}(t)$, that will give the product operational matrix of the shifted Legendre basis. First, we write

$$
\mathcal{L}_{i}(t) \mathcal{L}_{j}(t)=\sum_{r=0}^{i+j} a_{r} \mathcal{L}_{r}(t)
$$

The coefficients $a_{r}, r=0,1, \cdots, i+j$, are computed in the following manner. Multiplying both sides of equation (3.6) by $\mathcal{L}_{m}(t), m=0,1, \cdots, i+j$, and integrating the result yields

$$
\int_{0}^{1} \mathcal{L}_{i}(t) \mathcal{L}_{j}(t) \mathcal{L}_{m}(t) d t=\sum_{r=0}^{i+j} a_{r} \int_{0}^{1} \mathcal{L}_{r}(t) \mathcal{L}_{m}(t) d t=\frac{1}{2 m+1} a_{m} .
$$


Using (3.7) and the analytic form of the SLPs given by (3.1), we obtain

$$
\begin{aligned}
a_{m} & =(2 m+1) \int_{0}^{1} \mathcal{L}_{i}(t) \mathcal{L}_{j}(t) \mathcal{L}_{m}(t) d t \\
& =(2 m+1) \sum_{k=0}^{i} \sum_{l=0}^{j} \sum_{s=0}^{m} c_{i, k} c_{j, l} c_{m, s} \int_{0}^{1} t^{k+l+s} d t=(2 m+1) \Delta_{i, j, m},
\end{aligned}
$$

where

$$
\Delta_{i, j, m}=\sum_{k=0}^{i} \sum_{l=0}^{j} \sum_{s=0}^{m} \frac{c_{i, k} c_{j, l} c_{m, s}}{k+l+s+1} .
$$

By substituting $a_{m}$ into equation (3.6), we have

$$
\mathcal{L}_{i}(t) \mathcal{L}_{j}(t)=\sum_{m=0}^{i+j}(2 m+1) \Delta_{i, j, m}
$$

Suppose that

$$
\mathcal{C}=\left[\mathfrak{c}_{0}, \mathfrak{c}_{1}, \cdots, \mathfrak{c}_{M}\right]^{T},
$$

then using (3.8), we can write

$$
\mathfrak{L}(t) \mathfrak{L}^{T}(t) \mathcal{C} \simeq \overline{\mathcal{C}} \mathfrak{L}(t),
$$

where $\overline{\mathcal{C}}$ is an $(M+1) \times(M+1)$ matrix which is called the product operational matrix of the SLPs basis vector, and is given by

$$
\overline{\mathcal{C}}=\left[\bar{c}_{i, j}\right], \quad i, j=0,1, \cdots, M,
$$

where

$$
\bar{c}_{i, j}=\frac{2 j+1}{T} \sum_{m=0}^{M} \Delta_{i, j, m} \mathfrak{c}_{m} .
$$

Also, for an $(M+1) \times(M+1)$ matrix $D=\left[d_{i, j}\right], i, j=0,1, \cdots, M$, we have

$$
\mathfrak{L}^{T}(t) D \mathfrak{L}(t) \simeq D^{*} \mathfrak{L}(t),
$$

where $D^{*}=\left[d_{i}^{*}\right], i=0,1, \cdots, M$ is an $1 \times(M+1)$ vector given by

$$
d_{i}^{*}=(2 i+1) \sum_{m=0}^{M} \sum_{n=0}^{M} \Delta_{m, n, i} d_{m, n} .
$$

(3) The operational matrix of Rl-integral of order $\omega$ for vector $\mathfrak{L}(t)$ is as

$$
{ }^{R L} I_{t}^{\omega} \mathfrak{L}(t) \simeq \Upsilon^{\omega} \mathfrak{L}(t)
$$

where

$$
\Upsilon^{\omega}=\left[\sigma_{i, j}\right], \sigma_{i, j}=\sum_{p=0}^{i} \rho_{p, j} a_{i, p} \frac{\Gamma(p+1)}{\Gamma(p+\omega+1)}, \quad i, j=0,1, \ldots, M,
$$

with

$$
\rho_{p, j}=(2 j+1) \sum_{l=0}^{j} \frac{(-1)^{j+l}(j+l) !}{(j-l)(l !)^{2}(p+l+\omega+1)} .
$$


The matrix $\Upsilon^{\omega}$ is called the operational matrix of RL-integral based on the SLPs [2] .

(4) The operational matrix of AB-integral of order $\omega$ for vector $\mathfrak{L}(t)$ is obtained as

$$
{ }^{A B} I_{t}^{\omega} \mathfrak{L}(t)=\alpha_{\omega} \mathfrak{L}(t)+\beta_{\omega} \Gamma(\omega+1)^{R L} I_{t}^{\omega} \mathfrak{L}(t) .
$$

According to (3.11), we have

$$
\begin{aligned}
{ }^{A B} I_{t}^{\omega} \mathfrak{L}(t) & \simeq \alpha_{\omega} \mathfrak{L}(t)+\beta_{\omega} \Gamma(\omega+1) \Upsilon^{\omega} \mathfrak{L}(t) \\
& =\left(\alpha_{\omega} I+\beta_{\omega} \Gamma(\omega+1) \Upsilon^{\omega}\right) \mathfrak{L}(t)=J^{\omega} \mathfrak{L}(t),
\end{aligned}
$$

where $I$ is an $(M+1) \times(M+1)$ identity matrix and $J^{\omega}=\alpha_{\omega} I+\beta_{\omega} \Gamma(\omega+$ 1) $\Upsilon^{\omega}$. The matrix $J^{\omega}$ is called the operational matrix of AB-integral based on the SLPs.

\section{Numerical method}

In this section, we introduce a numerical method for the solution of nonlinear Volterra integro-differential equations of the form equation (1.1). For this suppose, first we approximate ${ }^{A B C} D_{t}^{\omega} u(t)$ as

$$
{ }^{A B C} D_{t}^{\omega} u(t) \simeq U^{T} \mathfrak{L}(t) .
$$

By taking the AB-integral of (4.1) and using initial condition, we have

$$
u(t) \simeq U^{T} J^{\omega} \mathfrak{L}(t)+u_{0} .
$$

We approximate $u_{0}$ as

$$
u_{0} \simeq \mathcal{U}^{T} \mathfrak{L}(t)
$$

By putting (4.3) in (4.2), we can write (4.2) as

$$
u(t) \simeq \gamma \mathfrak{L}(t),
$$

where $\gamma=U^{T} J^{\omega}+\mathcal{U}^{T}$. Let $g(t)=f(t, u(t))$ and $h(t)=\phi(t, u(t))$. Then we approximate $g(t)$ as

$$
g(t) \simeq G^{T} \mathfrak{L}(t)
$$

For approximating $T u(t)$, first $K(t, s)$ and $h(t)$ are approximated as

$$
\begin{aligned}
& K(t, s) \simeq \mathfrak{L}^{T}(t) \mathcal{K} \mathfrak{L}(s), \\
& h(t) \simeq H^{T} \mathfrak{L}(t) .
\end{aligned}
$$

By using (4.6) and (4.7), Tu(t) is approximated as

$$
\begin{aligned}
T u(t) & \simeq \int_{0}^{t} \mathfrak{L}^{T}(t) \mathcal{K} \mathfrak{L}(s) \mathfrak{L}^{T}(s) H d s \\
& =\mathfrak{L}^{T}(t) \mathcal{K} \int_{0}^{t} \mathfrak{L}(s) \mathfrak{L}^{T}(s) H d s \\
& =\mathfrak{L}^{T}(t) \mathcal{K} \int_{0}^{t} \bar{H} \mathfrak{L}(s) d s \\
& =\mathfrak{L}^{T}(t) \mathcal{K} \bar{H} \int_{0}^{t} \mathfrak{L}(s) d s=\mathfrak{L}^{T}(t) \mathcal{K} \bar{H} P \mathfrak{L}(s) .
\end{aligned}
$$


Substituting (4.1), (4.5) and (4.8) in equation (1.1), we have

$$
U^{T} \mathfrak{L}(t)=G^{T} \mathfrak{L}(t)+\mathfrak{L}^{T}(t) \mathcal{K} \bar{H} P \mathfrak{L}(s) .
$$

Let $\Lambda=\mathcal{K} \bar{H} P$. Using (3.10) to define the vector $\Lambda^{*}$, and using this approximation in (4.9) we obtain the following system

$$
U^{T}-G^{T}-\Lambda^{*}=0 .
$$

In veiw of (4.4), we rewrite (4.5) and (4.7) as

$$
\begin{aligned}
& f(t, \gamma \mathfrak{L}(t))=G^{T} \mathfrak{L}(t), \\
& \phi(t, \gamma \mathfrak{L}(t))=H^{T} \mathfrak{L}(t) .
\end{aligned}
$$

By putting $M+1$ points $t_{i}, i=1,2, \ldots, M+1$ in equation (4.11), gives

$$
\begin{gathered}
f\left(t_{i}, \gamma \mathfrak{L}\left(t_{i}\right)\right)-G^{T} \mathfrak{L}\left(t_{i}\right)=0, \\
\phi\left(t_{i}, \gamma \mathfrak{L}\left(t_{i}\right)\right)-H^{T} \mathfrak{L}\left(t_{i}\right)=0,
\end{gathered}
$$

where $t_{i}=\frac{i}{M+2}$.

Equations (4.10) and (4.12) form a system of $3(M+1)$ nonlinear equations of the vectors of $U, G$ and $H$. By solving this system, the unknown parameters of the vectors of $U, G$ and $H$ are obtained. Finally the approximate solution can be computed by (4.4).

\section{Error estimation}

In this section, we give an estimate for the error of the numerical solution of equation (1.1) with initial condition obtained by the proposed method in Section 4.

Theorem 5.1. Suppose that $u$ and $u_{M}$ satisfy the assumptions of Theorems 2.1 and 2.2. Assume that $u \in C^{M+1}[0,1]$ is the exact solution of equation (1.1) and $u_{M}(t)=\gamma^{T} \mathfrak{L}(t)$ is its approximation given by the method proposed in Section 4. Then, we have

$$
\begin{aligned}
& \left\|u(t)-u_{M}(t)\right\|_{2} \leq \frac{\rho}{(M+1) ! 2^{2 M+1}}, \\
& \left\|^{A B C} D_{t}^{\omega} u(t)-{ }^{A B C} D_{t}^{\omega} u_{M}(t)\right\|_{\infty} \leq \frac{\delta \rho}{\sqrt{M+1} M ! 2^{2 M+1}}, \\
& \left\|^{A B} I_{t}^{\omega} u(t)-{ }^{A B} I_{t}^{\omega} u_{M}(t)\right\|_{\infty} \leq \frac{\varepsilon \rho}{\sqrt{M+1} M ! 2^{2 M+1}},
\end{aligned}
$$

where $\rho=\sup _{\theta \in[0,1]}\left|u^{(M+1)}(\theta)\right|$.

Proof. Let that $u^{*}(t)$ is the interpolating polynomials to $u(t)$ at points $t_{i}$, $i=0,1, \ldots, M$ are the roots of the shifted Chebyshev polynomials of degree $M+1$, then we can write

$$
u(t)-u^{*}(t)=\frac{u^{(M+1)}(\theta)}{(M+1) !} \prod_{i=0}^{M}\left(t-t_{i}\right),
$$

where $\theta \in[0,1]$. 
According to Chebyshev interpolation nodes, for $t \in[0,1]$ it gives

$$
\left|u(t)-u^{*}(t)\right|=\frac{\rho}{(M+1) ! 2^{2 M+1}},
$$

where $\rho=\sup _{\theta \in[0,1]}\left|u^{(M+1)}(\theta)\right|$.

Since the best approximation of a given function $u \in C^{M+1}[0,1]$ in the finite subspace $S_{M}=\left\{\mathcal{L}_{0}(t), \mathcal{L}_{1}(t), \ldots, \mathcal{L}_{M}(t)\right\}$ is unique, we can write

$$
\begin{aligned}
\left\|u(t)-u_{M}(t)\right\|_{2}^{2} & \leq\left\|u(t)-u^{*}(t)\right\|_{2}^{2} \\
& =\int_{0}^{1}\left|u(t)-u^{*}(t)\right|^{2} d t \\
& =\int_{0}^{1}\left(\frac{\rho}{(M+1) ! 2^{2 M+1}}\right)^{2} d t=\left(\frac{\rho}{(M+1) ! 2^{2 M+1}}\right)^{2} .
\end{aligned}
$$

Taking the squared root of both sides of (5.1) gets

$$
\left\|u(t)-u_{M}(t)\right\|_{2} \leq \frac{\rho}{(M+1) ! 2^{2 M+1}} .
$$

We know that $\|\cdot\|_{\infty} \leq \sqrt{M+1}\|\cdot\|_{2}$. Then, in veiw of Theorems 2.1 and 2.2 , the proof is complete.

\section{Test examples}

In this section, we consider several examples to confirm the accuracy and applicability of the proposed method.

Example 6.1. Let $f(t, u(t))=2 t-1-\frac{1}{4} t\left(-2 e+2 e^{1-t+t^{2}}+\sqrt{\pi} e^{\frac{3}{4}}\left(\operatorname{Er} f i\left[\frac{1}{2}\right]\right.\right.$ $\left.\left.-\operatorname{Erfi}\left[\frac{1}{2}-t\right]\right)\right), \lambda=1, u(0)=1$ and $T u(t)$ is given as

$$
T u(t)=\int_{0}^{t} t s e^{u(s)} d s .
$$

$\operatorname{Erfi}(\cdot)$ is the imaginary error function. The analytical solution of this example is $u(t)=1-t+t^{2}$ when $\omega=1$. We solved this example by the proposed method. By considering $M=5$, the numerical results are shown in Figure 1 by different values of $\omega$. As seen in Figure 1, when the value of $\omega$ tends to 1, the approximate solution converges to the analytical solution.

Example 6.2. Let $f(t, u(t))=e^{t}\left(1+e^{2}\right)-\frac{1}{4} t^{2}\left(1+e^{2 t}(-1+2 t)\right)-u^{2}(t)$, $\lambda=1, u(0)=1$ and $T u(t)$ is given as

$$
T u(t)=\int_{0}^{t} t^{2} s u^{2}(s) d s .
$$

The analytical solution of this example is $u(t)=e^{t}$ when $\omega=1$. This example is solved by the proposed method with different values of $\omega$. By considering $M=5$, the numerical results are plotted in Figure 2.

Example 6.3. Let $f(t, u(t))=1-u^{2}(t), \lambda=0$ and $u(0)=0$. The analytical solution of this example is $u(t)=\frac{e^{2 t}-1}{e^{2 t}+1}$ when $\omega=1$. By applying the proposed method, we solved this example. By considering $M=5$, the numerical results are 


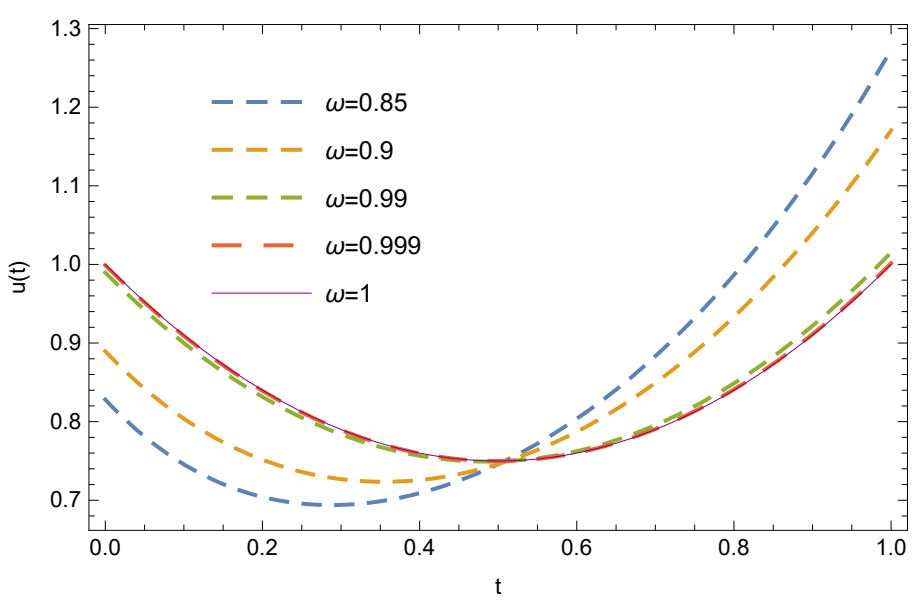

Figure 1. (Example 6.1) The exact and approximate solutions given by different values of $\omega$ and $M=5$.

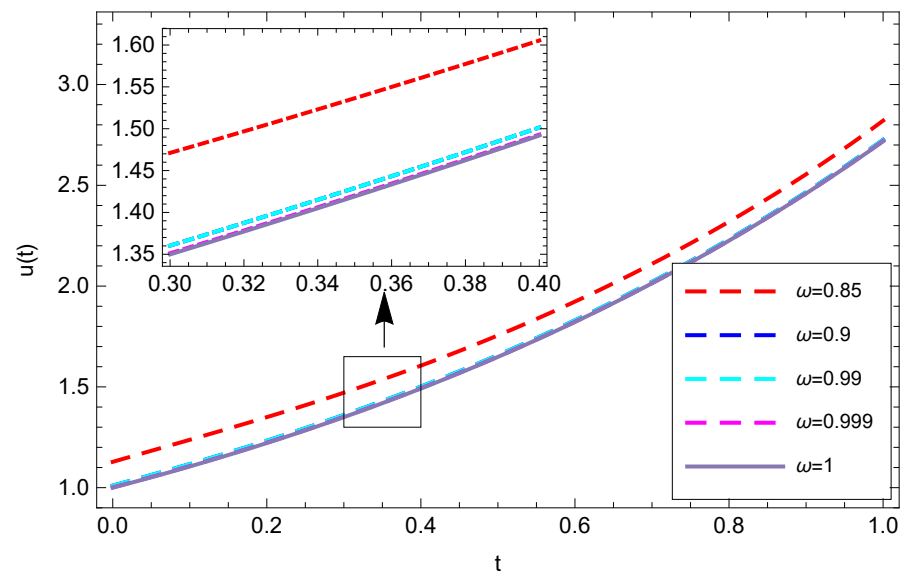

Figure 2. (Example 6.2) The exact and approximate solutions given by different values of $\omega$ and $M=5$.

plotted in Figure 3 by different values of $\omega$. As seen in Figure 3, when the value of $\omega$ tends to 1, the approximate solution converges to the analytical solution.

Example 6.4. Consider the following system of nonlinear Volterra integro-differential equation

$$
\left\{\begin{array}{l}
{ }_{A B C} D^{\omega} u(t)=f_{1}(t, u(t), v(t))+\lambda_{1} T_{1}(u, v)(t) \\
{ }^{A B C} D^{\omega} v(t)=f_{2}(t, u(t), v(t))+\lambda_{2} T_{2}(u, v)(t)
\end{array}\right.
$$




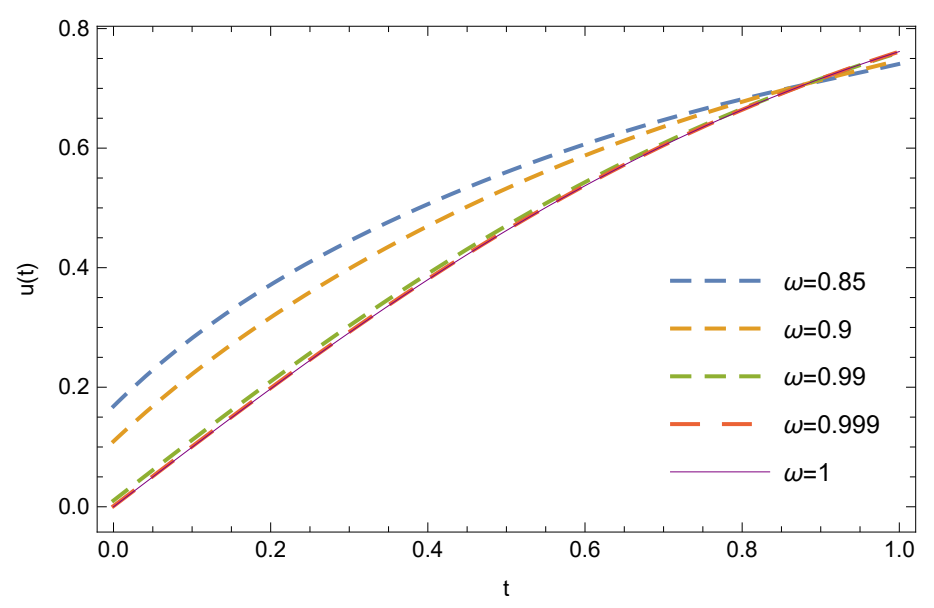

Figure 3. (Example 6.3) The exact and approximate solutions given by different values of $\omega$ and $M=5$.

where

$$
\left\{\begin{array}{l}
\lambda_{1}=-1, \quad \lambda_{2}=1 \\
f_{1}(t, u(t), v(t))=1+t^{3}+t^{4} \\
f_{2}(t, u(t), v(t))=1-\frac{t^{3}}{2}-\frac{t^{4}}{3} \\
T_{1}(u, v)(t)=\int_{0}^{t} t^{2}(u(s)+v(s)) d s \\
T_{2}(u, v)(t)=\int_{0}^{t} t u(s) v(s) d s \\
u(0)=0, \quad v(0)=1 .
\end{array}\right.
$$

The analytical solutions of this example are $u(t)=t$ and $v(t)=1+t$ when $\omega=1$. By using the proposed method, we solved this example. By considering $M=5$, the numerical results are reported in Figure 4, Table 1 and 2 by different values of $\omega$. The exact and numerical solutions obtained by different values of $\omega$ are plotted in Figure 4. Furthermore, in Table 1 and 2, the exact and numerical solutions at some grid points with different values of $\omega$ are seen.

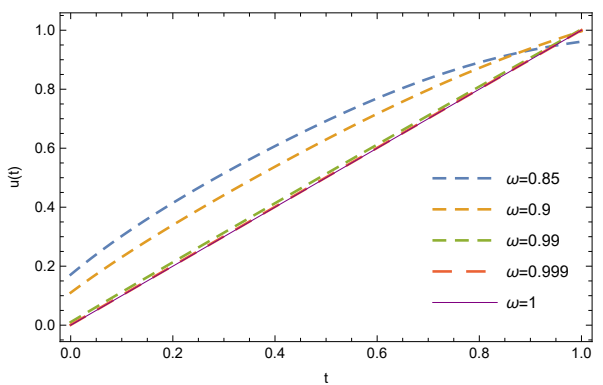

(a)

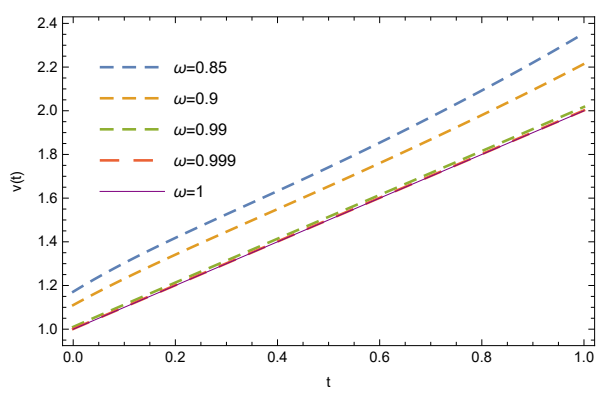

(b)

Figure 4. (Example 6.4) (a) The exact and approximate solutions $(u(t))(\mathrm{b})$ The exact and approximate solutions $(v(t))$ and $M=5$. 
TABLE 1. (Example 6.4) Numerical results $u(t)$ by different values of $\omega$ and $M=5$.

\begin{tabular}{|c|c|c|c|c|c|}
\hline$t$ & $\omega=0.85$ & $\omega=0.9$ & $\omega=0.99$ & $\omega=0.999$ & $\omega=1$ \\
\hline 0.1 & 0.30231 & 0.23076 & 0.11235 & 0.10123 & 0.10000 \\
0.3 & 0.51480 & 0.44093 & 0.31363 & 0.30136 & 0.30000 \\
0.5 & 0.69220 & 0.62946 & 0.51305 & 0.50131 & 0.50000 \\
0.7 & 0.83586 & 0.79789 & 0.71085 & 0.70109 & 0.70000 \\
0.9 & 0.93217 & 0.93812 & 0.90640 & 0.90066 & 0.90000 \\
\hline
\end{tabular}

TABLE 2. (Example 6.4) Numerical results $v(t)$ by different values of $\omega$ and $M=5$.

\begin{tabular}{|c|c|c|c|c|c|}
\hline$t$ & $\omega=0.85$ & $\omega=0.9$ & $\omega=0.99$ & $\omega=0.999$ & $\omega=1$ \\
\hline 0.1 & 1.30285 & 1.23099 & 1.11236 & 1.10123 & 1.10000 \\
0.3 & 1.52586 & 1.44615 & 1.31384 & 1.30138 & 1.30000 \\
0.5 & 1.74109 & 1.65396 & 1.51429 & 1.50142 & 1.50000 \\
0.7 & 1.97079 & 1.86860 & 1.71497 & 1.70148 & 1.70000 \\
0.9 & 2.22022 & 2.09479 & 1.91644 & 1.90162 & 1.90000 \\
\hline
\end{tabular}

\section{Conclusion}

In this work, we presented a numerical method based on operational matrices to solve Volterra integro-differential equations. For this do, we used the operational matrixces of integral, produce, fractional integral of Riemann-Liouville type and obtained the operational matrix of fractional integral of Atangana and Baleanu type. Then, by substituting these matrices and collocation points, we converted the Volterra integro-differential equations to an algebraic system. By solving this system, we obtained the approximate solution. Finally, some examples are considered to confirm the accuracy of the proposed method.

\section{References}

[1] A. Atangana and D. Baleanu, New fractional derivatives with nonlocal and nonsingular kernel: theory and application to heat transfer model, Thermal Science, $\mathbf{2 0}$ (2) (2016), 763-769.

[2] A. H. Bhrawy, M. A. Zaky and R. A. Van Gorder, A space-time Legendre spectral tau method for the two-sided space-time Caputo fractional diffusion-wave equation, Numer. Algorithms, 71 (1) (2016), 151-180.

[3] M. Caputo and M. Fabrizio, A new definition of fractional derivative without singular kernel, Progress in Fractional Differentiation and Applications, 1 (2) (2015), 73-85.

[4] W. Chen, A speculative study of 2/3-order fractional Laplacian modeling of turbulence: some thoughts and conjectures, Chaos: An Interdisciplinary Journal of Nonlinear Science, 16 (2) (2006), 120-126.

[5] W. Chen, H. G. Sun, X. D. Zhang and D. Korosak, Anomalous diffusion modeling by fractal and fractional derivatives, Comput. Math. Appl., 59 (5) (2010), 1754-1758.

[6] D.B. Dhaigude and B.H. Rizqan, Existence and uniqueness of solutions for fractional differential equations with advanced arguments, Advanced Math. Models 86 Applications, 2(3) (2017), 240-250. 
[7] E. H. Doha, M. A. Abdelkawy, A. Z. M. Amin and D. Baleanu, Spectral technique for solving variable-order fractional Volterra integro-differential equations, Numer. Methods Partial. Differ. Equ., 34 (5) (2018), 1659-1677.

[8] A. A. El-Sayed and P. Agarwal, Numerical solution of multiterm variable-order fractional differential equations via shifted Legendre polynomials, Math. Methods Appl. Sci., 42 (11) (2019), 3978-3991.

[9] R. M. Ganji and H. Jafari, A numerical approach for multi-variable orders differential equations using Jacobi polynomials, International Journal of Applied and Computational Mathematics, 5 (4) (2019), 5:34.

[10] R. M. Ganji and H. Jafari, Numerical solution of variable order integro-differential equations, Advanced Mathematical Models and Applications, 4 (1) (2019), 64-69.

[11] R. M. Ganji, H. Jafari and A. R. Adem, A numerical scheme to solve variable order diffusion-wave equations, Thermal Science, (2019), 371-371.

[12] R. M. Ganji, H. Jafari and D. Baleanu, A new approach for solving multi variable orders differential equations with Mittag-Leffler kernel, Chaos, Solitons and Fractals, 130 (2020), 109405.

[13] V. Gill and K. Modi, Pathway fractional integral operator involving certain special functions, Advanced Math. Models \& Applications, 2(2) (2017), 88-96.

[14] A. Khalouta and A. Kadem, A new technique for finding exact solutions of nonlinear time-fractional wave-like equations with variable coefficients, Proceedings of the Institute of Mathematics and Mechanics, 45 (2) (2019), 167-180.

[15] A. A. Kilbas, H. M. Srivastava and J. J. Trujillo, Theory and Applications of Fractional Differential Equations, 204 (North-Holland Mathematics Studies), Elsevier Science Inc. New York, (2006)

[16] Z. Li, H. Wang, R. Xiao and S. Yang, A variable-order fractional differential equation model of shape memory polymers, Chaos, Solitons and Fractals, 102 (2017), 473485.

[17] J. Liu, H. Fu, H. Wang and X. Chai, A preconditioned fast quadratic spline collocation method for two-sided space-fractional partial differential equations, J. Comput. Appl. Math., 360 (2019), 138-156.

[18] J. Liu, X. Li and L. Wu, An operational matrix technique for solving variable order fractional differential-integral equation based on the second kind of chebyshev polynomials, Advances in Mathematical Physics, (2016), 1-9.

[19] X.J. Ma, H. M. Srivastava, D. Baleanu, and , X.J. Yang, A New Neumann Series Method for Solving a Family of Local Fractional Fredholm and Volterra Integral Equations, Math. Probl. Eng., 2013 (2013), (Article ID 325121):6.

[20] W. Malesza, M. Macias and D. Sierociuk, Analytical solution of fractional variable order differential equations, J. Comput. Appl. Math., 348 (2019), 214-236.

[21] M. J. Mardanov, Y. A. Sharifov and F. M. Zeynalli, Existence And uniqueness of the solutions to impulsive nonlinear integro-differential equations with nonlocal boundary conditions, Proceedings of the Institute of Mathematics and Mechanics, 45 (2) (2019), 222-233.

[22] S. Nemati, P. M. Lima and Y. Ordokhani, Numerical solution of a class of twodimensional nonlinear Volterra integral equations using Legendre polynomials, $J$. Comput. Appl. Math., 242 (2013), 53-69.

[23] H. T. C. Pedro, M. H. Kobayashi, J. M. C. Pereira and C. F. M. Coimbra, Variable order modeling of diffusive-convective effects on the oscillatory flow past a sphere, IFAC Proceedings Volumes, 39 (11) (2006), 454-459.

[24] S. A. Rakhshan and S. Effati, A generalized Legendre-Gauss collocation method for solving nonlinear fractional differential equations with time varying delays, Appl. Numer. Math., 146 (2019), 342-360. 
[25] C. Ravichandran, K. Logeswari and Fahd Jarad, New results on existence in the framework of Atangana-Baleanu derivative for fractional integro-differential equations, Chaos, Solitons and Fractals, 125 (2019), 194-200.

[26] Y. A. Rossikhin and M. V. Shitikova, Application of fractional derivatives to the analysis of damped vibrations of viscoelastic single mass systems, Acta. Mech., 120 (1) (1997), 109-125.

[27] Y.A. Sharifov, F.M. Zeynally and S.M. Zeynally, Existence and uniqueness of solutions for nonlinear fractional differential equations with two-point boundary conditions, Advanced Math. Models \& Applications, 3(1) (2018), 54-62.

[28] H. Singh, D. Baleanu, H. M. Srivastava, H. Dutta and N. Kumar Jha, Solution of multi-dimensional Fredholm equations using Legendre scaling functions, Appl. Numer. Math. 150 (2020), 313-324.

[29] H. G. Sun, W. Chen and Y. Q. Chen, Variable-order fractional differential operators in anomalous diffusion modeling, Physica A: Statistical Mechanics and its Applications, 388 (21) (2009), 4586-4592.

[30] X. J. Yang, General Fractional Derivatives: Theory, Methods and Applications, New York: CRC Press, 2019.

[31] X. J. Yang and J. A. Tenreiro Machado, A new fractional operator of variable order: application in the description of anomalous diffusion, Physica A: Statistical Mechanics and its Applications, 481 (2017), 276-283.

[32] F. Zakes and P. Sniady, Application of Volterra integral equations in dynamics of multispan uniform continuous beams subjected to a moving load, Shock and Vibration, (2016), 1-12.

[33] M. Zhao and H. Wang, Fast finite difference methods for space-time fractional partial differential equations in three space dimensions with nonlocal boundary conditions, Appl. Numer. Math., 145 (2019), 411-428.

Roghayeh Moallem Ganji

Department of Mathematics, Faculty of Mathematical Sciences, University of Mazandaran, Babolsar, Iran.

E-mail address: r.moallem.ganji@gmail.com

Hossein Jafari

Department of Mathematics, Faculty of Mathematical Sciences, University of Mazandaran, Babolsar, Iran.

Department of Mathematical sciences, University of South Africa, UNISA0003, South Africa.

E-mail address: jafari.usern@gmail.com

Received: December 24, 2019; Revised: April 12, 2020; Accepted: April 15, 2020 